\title{
Background reduction by a getter pump around the ionization volume of a Lamb-shift polarimeter and possible improvements of polarized ion sources
}

\author{
R. Engels ${ }^{a)}$ \\ Institut für Kernphysik, Forschungszentrum Jülich, Leo-Brandt-Strasse 1, 52425 Jülich, Germany \\ R. Emmerich \\ Institut für Kernphysik der Universität zu Köln, Zülpicher Strasse 77, 50937 Köln, Germany \\ K. Grigoryev ${ }^{\text {b) }}$ \\ Institut für Kernphysik, Forschungszentrum Jülich, Leo-Brandt-Strasse 1, 52425 Jülich, Germany \\ H. Paetz gen. Schieck and J. Ley \\ Institut für Kernphysik der Universität zu Köln, Zülpicher Strasse 77, 50937 Köln, Germany \\ M. Mikirtychyants \\ St. Petersburg Nuclear Physics Institute, Orlova Rosha 2, 188300 Gatchina, Russia \\ F. Rathmann, J. Sarkadi, and H. Seyfarth \\ Institut für Kernphysik, Forschungszentrum Jülich, Leo-Brandt-Strasse 1, 52425 Jülich, Germany \\ G. Tenckhoff \\ Institut für Kernphysik der Universität zu Köln, Zülpicher Strasse 77, 50937 Köln, Germany
}

\author{
A. Vasilyev \\ St. Petersburg Nuclear Physics Institute, Orlova Rosha 2, 188300 Gatchina, Russia
}

(Received 12 January 2005; accepted 7 March 2005; published online 26 April 2005)

\begin{abstract}
The Köln-Jülich Lamb-shift polarimeter is used to measure the nuclear polarization of the hydrogen or deuterium beam produced with the atomic-beam source for the polarized target at the ANKE spectrometer at COSY-Jülich. The precision of the earlier results had been dominated by the recombination of atoms in the ionizer. Protons or deuterons from the dissociative ionization of unpolarized recombined $\mathrm{H}_{2}$ or $\mathrm{D}_{2}$ molecules had strongly contributed to the extracted ion beam. To suppress this effect, in the new ionizer a nonevaporable getter pump of about $20001 / \mathrm{s} \mathrm{H}_{2}$ or $\mathrm{D}_{2}$ pumping speed surrounds the ionization volume. It reduces the extracted current of unpolarized ions, produced from the recombined molecular gas, by a factor of about 20 compared with the earlier value, which reduces the error of the polarization measurements to about $0.5 \%$. Now the $\mathrm{H}_{2}$ or $\mathrm{D}_{2}$ molecules in the ionization volume predominantly are those which are contained in the incoming beam from the atomic beam source. This allows the measurement of the fraction of unpolarized molecules in the polarized atomic $\overrightarrow{\mathrm{H}}$ or $\overrightarrow{\mathrm{D}}$ beam. The improvement achieved is a valuable step toward the measurement of the nuclear polarization of a gas sample, extracted from the storage cell of the polarized internal gas target for the spectrometer ANKE in the COSY-Jülich storage ring with the Lamb-shift polarimeter. Furthermore, the results show that the polarization of proton or deuteron beams would be increased by the installation of such a pump around the ionization volume of atomic-beam ion sources with an electron-impact ionizer. For ECR ionizers the recombined $\mathrm{H}_{2}$ or $\mathrm{D}_{2}$ molecules would be absorbed, whereas the noble gases, used as buffer, are not pumped by the getter material. (C) 2005 American Institute of Physics. [DOI: 10.1063/1.1898923]
\end{abstract}

\section{INTRODUCTION}

To produce polarized proton or deuteron beams, atoms of polarized atomic hydrogen or deuterium beams are ionized in various types of ionizers. However, the polarization of the extracted ion beam is always lower than the nuclear polarization of the primary atomic beam. For example, the

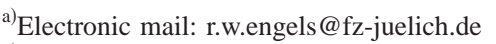

${ }^{b)} \mathrm{Ph} . D$. student from St. Petersburg Nuclear Physics Insitute, Orlova Rosha 2; 188300 Gatchina, Russia.
}

nuclear polarization of a hydrogen beam, produced with an atomic beam source (ABS) and injected into the ionizer, can be higher than $0.95 .{ }^{1}$ Even for such a high value, the polarization of the proton beam, extracted from the ionizer, may be 0.75 only. $^{2}$ The loss of polarization is mainly caused by recombination of atoms in the ionizer. Even if only a small fraction of the atoms of the incoming beam recombines in the ionizer, due to the complex structure around the ionization volume the molecules are stored and their partial pressure can increase by orders of magnitude compared to the 


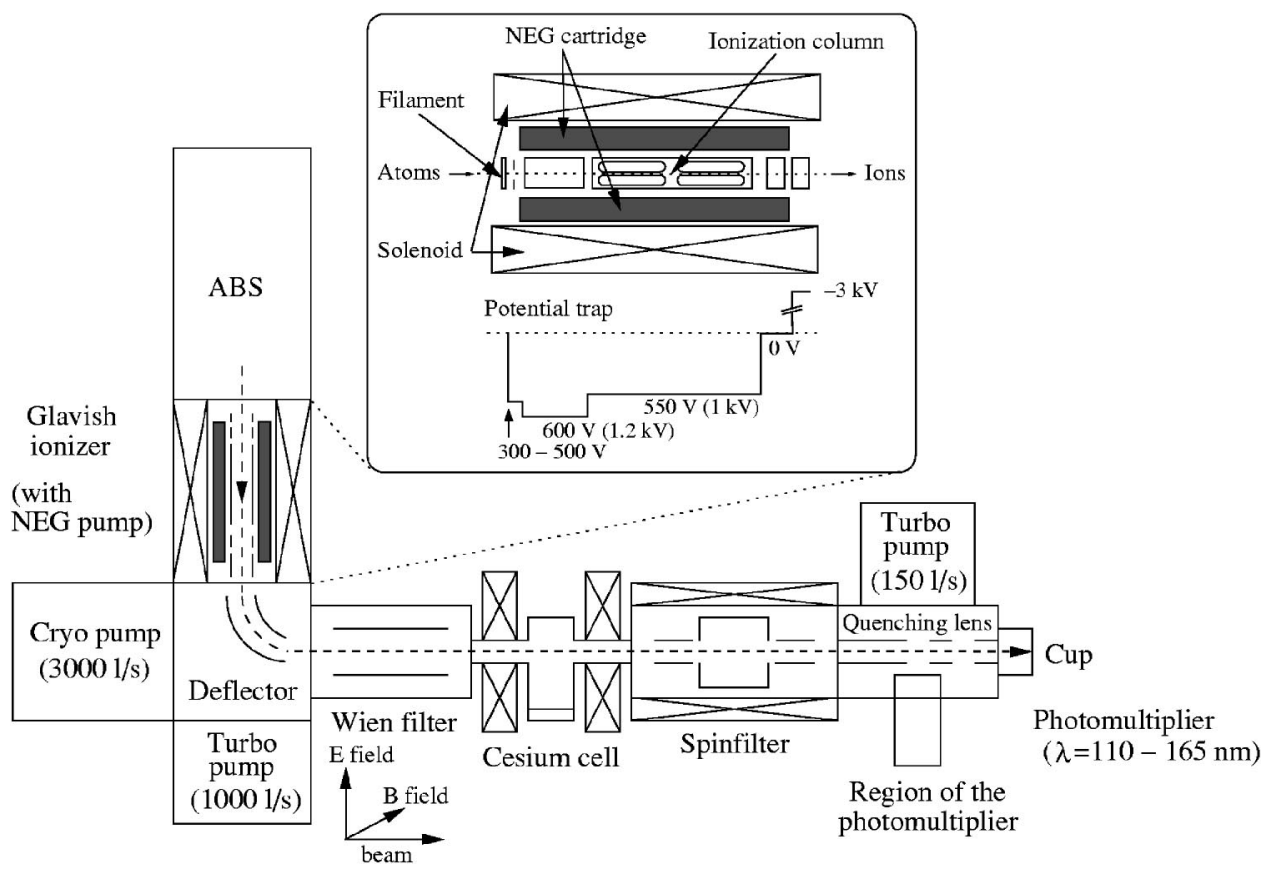

FIG. 1. Scheme of vertical polarized atomic beam source and ionizer (with the NEG pump of the new ionizer indicated in black), $90^{\circ}$ deflector, and the horizontal components of the Lamb-shift polarimeter. residual-gas pressure without beam. After a high number of wall collisions, the molecules are unpolarized to a high degree. ${ }^{3}$ From them, the ionizers produce unpolarized ions. These cannot be separated from the polarized protons or deuterons and they either decrease the degree of polarization of the beam from the polarized ion source or they necessitate corrections, if the extracted ion beam is used to determine the nuclear polarization of the primary atomic beam. Thus, a reduction of the contribution by recombined molecules is highly desirable.

Space limitations like that by the solenoid, needed to produce the strong magnetic field for the ionizer, prohibit the installation of turbo or cryo pumps on the ionizer chamber. Furthermore, their pumping speed would be strongly reduced by the complicated structure of the components inside the ionizer. A means to reduce the pressure in the ionizer has been found by installing a nonevaporable getter (NEG) pump inside the ionizer around the ionization volume. NEG components with a total pumping speed of about $2000 \mathrm{l} / \mathrm{s}$ for $\mathrm{H}_{2}$ and $\mathrm{D}_{2}$ were implemented in the new ionizer of the KölnJülich Lamb-shift polarimeter (LSP), ${ }^{4}$ which is used for tuning the high-frequency transition units of the ABS and which will be used to measure the nuclear polarization of the target gas in the storage cell of the polarized internal target ${ }^{5}$ for the magnet-spectrometer setup ANKE at COSY-Jülich.

\section{THE IONIZER AS A COMPONENT OF THE LAMB- SHIFT POLARIMETER}

The whole setup with the ABS, which delivers the vertical polarized $\overrightarrow{\mathrm{H}}$ or $\overrightarrow{\mathrm{D}}$ beam, the electrostatic $90^{\circ}$ deflector, and the LSP to measure the nuclear polarization of the beam, is shown in Fig. 1. The LSP consists of

(1) the Glavish-type ${ }^{6}$ ionizer,

(2) the Wien filter to re-establish longitudinal polarization after the deflection and to provide mass separation,
(3) the Cs vapor charge-exchange cell to produce metastable atoms from the ions,

(4) the spin filter to select metastable atoms in the different hyperfine states,

(5) the chamber for Stark quenching and Lyman $\alpha$ light measurement.

In the Glavish (low potential) ionizer mode, the atoms from the ABS are ionized by electrons emitted from a filament and get accelerated into the ionization volume to about $500 \mathrm{eV}$. There they are trapped by the potentials, applied to a system of cylindrical electrodes, which are also used to extract and to focus the produced ions. The gyration of the electrons in the longitudinal magnetic field, needed to decouple the electron and nuclear magnetic moments in the ionization process, increases the ionization probability by orders of magnitude to about $10^{-4}$.

In the earlier version of the ionizer, vacuum pumps could only be mounted on the chambers adjacent to the ionizer chamber. The solenoidal coil prevents the installation of pumps on the ionizer chamber itself. The complex structure of the components inside the ionizer chamber leads to a severe reduction of the pumping speed for atoms, scattered off the beam, and recombined molecules. Molecules, scattered back into the ionization volume and ionized there, have undergone a high number of wall collisions and they can be assumed to have lost their polarization. Thus, the contribution by the products of their dissociative ionization to the extracted ion beam is unpolarized. The protons or deuterons from recombined molecules in the extracted beam cannot be separated from those which directly originate from the polarized atoms in the beam. They contribute by equal parts to the peaks in the Lyman- $\alpha$ spectra measured with the photomultiplier at the quench chamber. The ratios of the peaks in these spectra yield the nuclear polarization of the $\vec{H}$ or $\vec{D}$ beam from the ABS. Equally distributed contributions to the peaks diminish the peak ratios and necessitate corrections. 


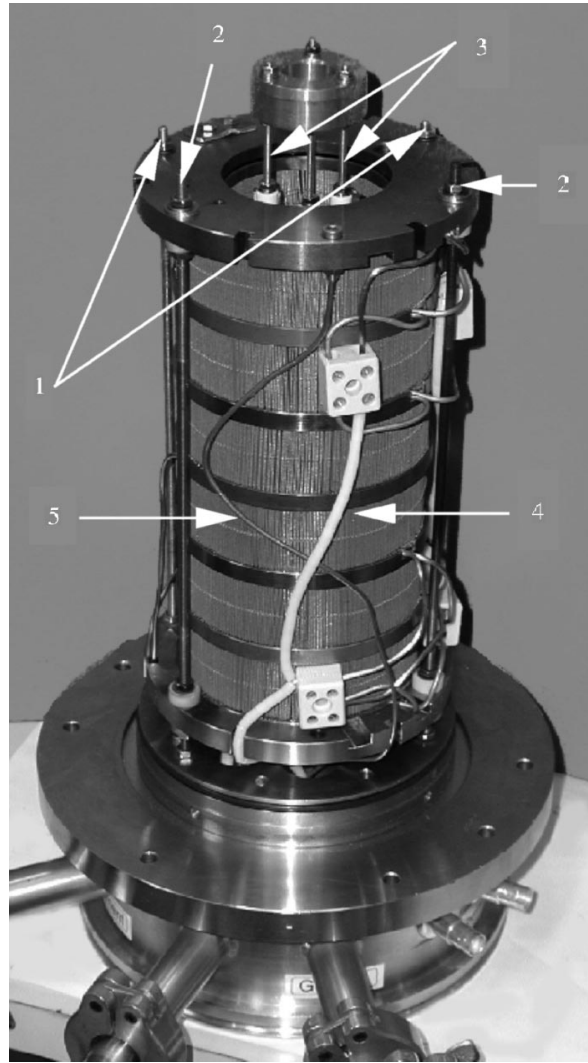

FIG. 2. The nonevaporable getter-pump assembly.

It was shown in Ref. 4 that the effect by protons from the residual gas in the ionizer chamber, also leading to an unpolarized backgound in the measured peaks, can be determined by spectra taken with the ABS beam switched off. For the typical residual gas pressure in the ionizer chamber of about $10^{-8}$ mbar, the resulting correction factor is small and lies around 1.005. No such contribution exists for deuterium, because the amount of deuterated residual gas molecules can be neglected.

The effect by recombined $\mathrm{H}_{2}$ molecules has been studied ${ }^{4}$ by admission of varying amounts of $\mathrm{H}_{2}$ into the ionizer chamber, i.e., by varying the equilibrium $\mathrm{H}_{2}$ pressure. With use of the Wien filter, the ratio of the extracted proton current from dissociative ionization to that of $\mathrm{H}_{2}^{+}$from ionization was measured to have a constant value $r_{1}$ $=0.095 \pm 0.008$ for $\mathrm{H}_{2}$ pressures up to $2 \times 10^{-6}$ mbar. With the assumption of equilibrium distribution of the recombined $\mathrm{H}_{2}$ gas in the ionizer chamber, this value leads to a correction factor $C_{\mathrm{rec}}=1.095 \pm 0.008$ to be applied to the measured Lyman- $\alpha$ peak ratio for hydrogen. With the previous ionizer, this correction was the main limitation of the accuracy in the determination of the nuclear polarization of the $\vec{H}$ or $\vec{D}$ beam from the ABS. Thus, suppression of the contribution by ions from recombined molecules was highly desirable.

\section{THE NEW IONIZER WITH A NONEVAPORABLE GETTER (NEG) PUMP AROUND THE IONIZATION VOLUME}

A new ionizer has been designed and built. Besides other minor modifications the essential improvements compared to

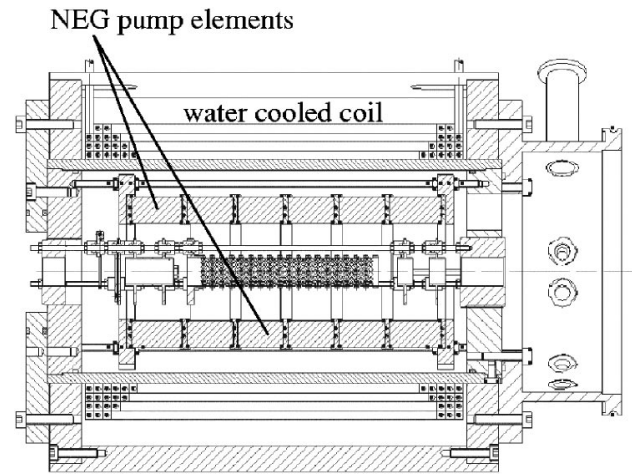

FIG. 3. Cut through the new ionizer with the six NEG elements. The inner free diameter of the electrodes and the mesh around the ionization volume is $15 \mathrm{~mm}$, the total length including the chamber at the exit flange is $37 \mathrm{~cm}$. The atomic beam enters from the left.

the earlier version are (i) installation of a NEG pump around the ionization volume and (ii) use of a water-cooled $\mathrm{Cu}$ hollow-conductor coil to produce a somewhat higher solenoidal magnetic field at an increased inner coil diameter.

Contrary to earlier binary getter alloys, ternary alloys have been found which can be operated at room temperature. Furthermore, they can be activated at lower temperatures. The getter alloys are commercially available as deposited on thin metallic strips. The NEG pump, installed in the new ionizer, is an assembly of six getter elements, taken from commercial cartridges. ${ }^{7}$ Each element consists of a thin, starshaped folded, 30-mm-wide amagnetic Constantan strip, covered on both sides by $70-\mu \mathrm{m}$-thick layers of getter material $(\mathrm{St} 707,70 \% \mathrm{Zr}, 24.6 \% \mathrm{~V}$, and $5.4 \% \mathrm{Fe})$. The free inner and the outer diameters are 60 and $98 \mathrm{~mm}$. The elements are fixed between home-made stainless-steel disks with circular grooves for the heating cables. ${ }^{8}$ During activation, the temperature of the getter elements is measured by $3 \mathrm{NiCr} / \mathrm{Ni}$ thermocouples, ${ }^{9}$ fixed to the central and the two outer disks. The photograph in Fig. 2 shows the pumping setup, mounted on the exit flange of the ionizer chamber, which also carries the chamber with the necessary feedthroughs. Figure 2 shows the two threaded rods (labeled 1) which clench the two confining rings toward the getter elements and the disks between them. Three additional threaded rods (two of them visible, label 2) are used to fix the assembly between the two end-flanges of the chamber as can be seen from the technical drawing of Fig. 3. The ceramic washers are installed to reduce the heat loss from the getter elements during regeneration. The ring at the upper end, fixed to an additional three inner threaded rods (label 3) serves for centering the system of electrodes, mounted on these rods as shown in Fig. 3, too. Furthermore, the photograph shows the supply lines to the heating cables (label 4) and the thermocouple lines (label 5). The whole setup is assembled outside and then introduced into the vacuum chamber. The outer diameter of the NEG assembly is kept as small as possible in order to minimize the inner diameter of the magnet coil. The current-supply unit, ${ }^{10}$ used to heat the getter elements for activation, is regulated by the control system of ABS and LSP. ${ }^{11}$

The pumping speed of a single getter element for $\mathrm{H}_{2}$ at room temperature and a pressure around $10^{-6}$ mbar is given 
by the producer as $475 \mathrm{l} / \mathrm{s}$, thus the total pumping speed would be $2850 \mathrm{l} / \mathrm{s}$. It is reduced, however, to about $2000 \mathrm{l} / \mathrm{s}$ by the closed star-shaped configuration around the inner volume. The total $\mathrm{H}_{2}$-sorption capacity at room temperature is given as 8100 Torr 1 .

The solenoidal coil is made from a $\mathrm{Cu}$ hollow-conductor of $0.5 \times 0.5 \mathrm{~cm}$ outer dimensions with a circular inner, water conducting tube of $0.3 \mathrm{~cm}$ diameter. Arranged in four layers, the coil windings cover a total length of $23.7 \mathrm{~cm}$. The inner coil diameter is $14.1 \mathrm{~cm}$. At the maximum possible current of $600 \mathrm{~A}$ it yields a magnetic flux density of about $0.5 \mathrm{~T}$.

\section{IMPROVEMENTS ACHIEVED BY THE GETTER PUMP}

The ions, extracted from the ionizer and deflected by $90^{\circ}$, in the setup of Fig. 1 are measured with the Faraday cup at the end of the LSP. The Wien filter is used to separate ions of different masses. The pressure in the ionizer chamber is measured with two hot-cathode gauges, ${ }^{12}$ installed in the adjacent ABS chamber and in the deflector chamber.

Before installation of the getter pump and with the ABS beam switched off, the residual gas pressure in the ionizer chamber was $1.5 \times 10^{-8}$ mbar. Electron-impact dissociative ionization of residual gas molecules like $\mathrm{H}_{2} \mathrm{O}$ yielded a proton current $I_{p}^{\text {off }}=10 \mathrm{nA}$. The current from single ionization only was $I_{\mathrm{H}_{2}^{+}}^{\text {off }}=5 \mathrm{nA}$. With the ABS beam switched on, the corresponding values were $I_{p}^{\mathrm{on}}=135 \mathrm{nA}$ and $I_{\mathrm{H}_{2}^{+}}^{\mathrm{on}}=160 \mathrm{nA}$. With the known ratio $r^{1}$ for the proton and the $\mathrm{H}_{2}^{+}$ion current from recombined molecules, the measured $\mathrm{H}_{2}^{+}$current yields a contribution of $15 \mathrm{nA}$ by protons from recombined molecules and leaves a current of $110 \mathrm{nA}$ by protons, produced by ionization of $\vec{H}$ atoms from the ABS beam. When the ionizer is used at higher potentials of more than $1000 \mathrm{~V}$, the efficiency is increased by one order and ion currents of $I_{p}^{\text {on }}$ $=1 \mu \mathrm{A}$ can be reached. But in this case the produced plasma is very sensitive to pressure changes and therefore, this plasma mode was not used for these measurements.

With the NEG pump installed and activated (heating to $400{ }^{\circ} \mathrm{C}$ for about $2 \mathrm{~h}$ ), the residual gas pressure is slightly reduced to $1.2 \times 10^{-8}$ mbar. Accordingly, the currents $I_{p}^{\text {off }}$ and $I_{\mathrm{H}_{2}^{+}}^{\text {off }}$ from the residual gas in the ionizer are reduced and within an accuracy of $0.2 \mathrm{nA}$ not measurable. The partial pressure of the water is especially reduced by heating of the getter pump and the pumping speed of the getter material has the highest value for $\mathrm{H}_{2}$ molecules.

A dramatic change is achieved in the current ratio with the ABS beam switched on. One now measures $I_{p}^{\text {on }}$ $=125 \mathrm{nA}$ and a strongly reduced current $I_{\mathrm{H}_{2}^{+}}^{\mathrm{on}}$ of only $7 \mathrm{nA}$ compared to the earlier value of $160 \mathrm{nA}$. The admixture of unpolarized protons to the extracted polarized beam is drastically reduced from the earlier $18 \%$ to $0.8 \%$.

The measurements of the continuous currents from the ionizer have been complemented by studies of the time dependence of these currents. The ABS beam was chopped by a rotating hollow cylinder of $56 \mathrm{~mm}$ outer diameter. Two opposite apertures, each extending over a fourth of the cylinder circumference, transmit the beam with a frequency

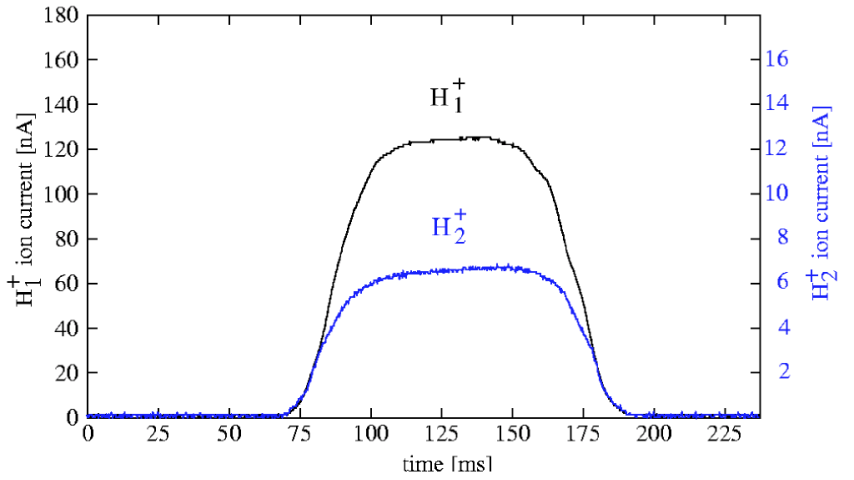

FIG. 4. The $\mathrm{H}_{1}^{+}$and the $\mathrm{H}_{2}^{+}$ion currents in the cup of the LSP as function of time with the ABS beam chopped.

twice the rotation frequency. A small aperture in a disk, rotating with the cylinder, induces a signal from a standard slotted optical CNY28 switch, which is used to trigger a storage oscilloscope. The chopper is installed in the lower ABS chamber in front of the sextupole magnets, which focus the beam into the ionizer. There the particle trajectories can be assumed to be roughly parallel and to cover the full magnet aperture diameter of $30 \mathrm{~mm}$. In this geometry and at a chopper-rotation frequency of $2 \mathrm{~Hz}$ one expects opening and closing times of $45 \mathrm{~ms}$ and intermediate $35 \mathrm{~ms}$ of full beam transmission. The measured time distributions agree with the expected behavior. An example is shown in Fig. 4. The slight increase of both currents during the phase of full transmission may indicate an asymmetry in the beam-density distribution or shows the residual increase of the $\mathrm{H}_{2}$ partial pressure after recombination of some hydrogen atoms to molecules. The measured current values agree with those obtained with the continuous beam.

\section{RESULTS AND EXPERIENCES ACHIEVED WITH THE NEW IONIZER}

With the previous ionizer, the ratio of the two peaks in the Lyman $\alpha$ spectrum had been measured as $0.780 \pm 0.002$ for the $\overrightarrow{\mathrm{H}}$ beam from the ABS with the atoms occupying the hydrogen hyperfine state $1 .{ }^{4}$ With the correction factor, $C_{\text {rec }}$ $=1.095 \pm 0.008$, taking into account the unpolarized protons from recombined molecules, and $C^{\prime}=1.016 \pm 0.004$, combining the other corrections, $p_{z}=0.867 \pm 0.009$ had been obtained for the nuclear polarization of the hydrogen beam from the ABS.

With the new ionizer and the NEG pump installed, but not activated, the Lyman $\alpha$ peak ratio was measured again as $0.780 \pm 0.003$. The correction factor $C_{\mathrm{rec}}=1.138 \pm 0.013$ for this case is higher than the earlier one, which is explained with the reduction of the external pumping speed by the narrow structure of the NEG pump. The coincidence of the measured peak ratio with the earlier value is an accidental one. With $C^{\prime}=1.016 \pm 0.004$ being unchanged, one now derives $p_{z}=0.901 \pm 0.013$. After activation of the NEG pump, $C_{\text {rec }}$ is strongly reduced to $1.0053 \pm 0.0004$ due to reduction of the background by unpolarized protons from recombined molecules. With this value and the unchanged $C^{\prime}$ one now obtains $p_{z}=0.898 \pm 0.004$, with a reduced error confirming 
the value obtained before NEG activation. Both values are slightly higher than the earlier one, possibly due to a higher degree of dissociation in the ABS beam. An alternative interpretation of the $\mathrm{H}_{2}^{+}$current, extracted from the ionizer, is given in the following.

One experience, made with the NEG pump, should be mentioned. Once, during heating for activation, it was realized that an additional component had to be installed at the ionizer chamber. Thus, the heating was stopped and the temperature of the NEG elements-at this time monitored only by two sensors at both ends of the pump-was observed until it dropped to room temperature, i.e., below the critical value of $50{ }^{\circ} \mathrm{C}$ given in the NEG manuals. Following the venting with air and opening of the chamber, the NEG-pump elements started to warm up to red heat. Immediate closing of the chamber and pumping could stop this process and avoid further damage. However, the NEG elements were destroyed and had to be replaced by a new set. Obviously, somewhere along the getter cartridge, the temperature locally had not dropped below the critical value and, starting in a confined region, the oxidation of the strip-coating getter material caused the spread of heating up. As mentioned earlier, now a third temperature sensor has been added at the center of the pumping setup to prevent such a failure in the future.

\section{APPLICATIONS BASED UPON THE IMPROVEMENT}

By the installation of the powerful NEG pump around the ionization volume of the ionizer, the contribution by protons and $\mathrm{H}_{2}^{+}$ions, resulting from recombined molecules, in the extracted beam from the ionizer, has been strongly suppressed. The achieved improvement of the ionizer performance allows a number of applications.

By introducing molecular $\mathrm{H}_{2}$ into the ionizer chamber, the ratio of the extracted currents has been measured as $I_{p} / I_{\mathrm{H}_{2}^{+}}=r_{1}=0.095 \pm 0.008$. The ratio agrees with values determined earlier, namely $\sim 0.11^{13}$ and $\sim 0.075$. $^{14}$ It can also be explained by the known electron-impact dissociative ionization cross section $\sigma_{\text {diss }}^{\text {ion }}($ Ref. 15) and the total ionization cross section $\sigma_{\text {tot }}^{\text {ion }}{ }^{16}$ For the full electron energy of $\sim 500 \mathrm{eV}$ (single ionization process) the ratio $\sigma_{\text {diss }}^{\text {ion }} / \sigma_{\text {tot }}^{\text {ion }}$ is $0.044 \pm 0.004$. The same value is obtained for averaging from $30 \mathrm{eV}$ to the full energy (multiple ionization processes). Good agreement with the present value of $0.095 \pm 0.008$ and the earlier current ratios $^{13,14}$ is achieved by doubling the cross-section ratio which indicates the dominance of the process $e+\mathrm{H}_{2} \rightarrow 3 e+2 \mathrm{H}^{+}$, i.e., double electron-impact ionization of $\mathrm{H}_{2}$ molecules in the ionization volume. The strongly reduced contribution by recombined molecules now suggests that the molecules are admixed to the $\overrightarrow{\mathrm{H}}$ beam, entering the ionizer from the ABS. Then the extracted ion currents $I_{p}$ $=125 \mathrm{nA}$ and $I_{\mathrm{H}_{2}^{+}}=(6.4 \pm 0.1) \mathrm{nA}$, measured with the ABS beam on, can be used to deduce an upper limit for the admixture of $\mathrm{H}_{2}$ molecules, entering the ionizer with the polarized atomic $\overrightarrow{\mathrm{H}}$ beam. The ratio of the ionization cross section of $\mathrm{H}_{2}$ to that of $\mathrm{H}$ for electrons of $\sim 500 \mathrm{eV}$ as well as for electron energies from the threshold energy to the accelera- tion energy is $r_{2}=1.7 \pm 0.1$. $^{16}$ With these data, the resulting degree of the admixture of $\mathrm{H}_{2}$ in the ABS beam is

$$
\frac{I_{\mathrm{H}_{2}}^{\mathrm{ABS}}}{I_{\mathrm{H}_{2}}^{\mathrm{ABS}}+I_{\mathrm{H}}^{\mathrm{ABS}}}=\frac{I_{\mathrm{H}_{2}^{+}}}{I_{\mathrm{H}_{2}^{+}}+r_{2} \cdot I_{p}-r_{1} \cdot I_{\mathrm{H}_{2}^{+}}}=0.029 \pm 0.004 .
$$

This value is derived under the assumption of equal efficiencies to extract protons and $\mathrm{H}_{2}^{+}$ions from the ionizer. However, it is confirmed by a measurement of the admixture using a crossed-beam quadrupole mass spectrometer, ${ }^{17}$ installed approximately at the ionizer position. A value of $0.033 \pm 0.007$ is measured in excellent agreement with that obtained from the currents extracted from the ionizer. For the experiments with the storage-cell gas target it is more convenient to use the degree of dissociation $\alpha$ of the beam, which gives the fraction of unpolarized protons injected in the $\mathrm{H}_{2}$ molecules. The two results for the $\mathrm{H}_{2}$ admixture yield

$$
\alpha=\frac{I_{\mathbf{H}}^{\mathrm{ABS}}}{I_{\mathbf{H}}^{\mathrm{ABS}}+2 \cdot I_{\mathrm{H}_{2}}^{\mathrm{ABS}}}=0.942 \pm 0.007,
$$

where the factor 2 in the denominator is due to the fact that, from a molecule, two unpolarized protons contribute to the beam and thus to the gas injected to the target storage cell.

Together with the ABS, which will feed the polarized gas target at the spectrometer ANKE, the LSP will be used to measure the nuclear polarization of the target gas at a gas sample, effusing from the target cell into the ionizer. The angular distribution of atoms and molecules will be much wider than that of the well collimated beam from the ABS. Thus, the fraction of particles, scattered by ionizer components, is expected to be essentially higher. The NEG pump around the ionization volume strongly reduces the background of unpolarized protons, extracted from the ionizer. With it, the measurements of the gas sample, necessitating the high sensitivity of the LSP, are thought feasible.

By installation of the NEG pump, the measured asymmetry of the peaks in the Lyman spectrum for a hydrogen beam from the ABS with the atoms in HFS 1 was increased from $0.780 \pm 0.002$ to $0.880 \pm 0.003$. Taking into account the correction factors for the spin filter, the cesium cell, and the Wien filter, ${ }^{4}$ the resulting polarizations $P_{p}$ of the proton beam, extracted from the ionizer, are $0.788 \pm 0.004$ without and $0.889 \pm 0.004$ with the NEG pump. With the measured proton beam intensities $I_{p}$, the figure of merit $P_{p}^{2} \cdot I_{p}$, measuring the proton-beam quality, is increased by about $23 \%$. The improvement of the beam quality can also be achieved for ECR ionizers because noble gases, used as buffer gas, are not pumped by the getter material. But one should be aware that, depending on the design of the individual ECR ionizer, the heating of the getter material during activation may destroy the magnetic properties of permanent magnets used for the ECR plasma.

\section{ACKNOWLEDGMENTS}

The authors wish to acknowledge the financial support by the German ministry BMBF (06 OK $862 \mathrm{I}$ and $06 \mathrm{OK}$ 958) and FZ Jülich. 
${ }^{1}$ M. Mikirtychyants et al., Proceedings of the Ninth International Workshop on Polarized Sources and Targets (PST01), Nashville, IN, 2001, edited by V. P. Derenchuk and B. Przewoski (World Scientific, 2002), p. 47.

${ }^{2}$ T. B. Clegg, Proceedings of the Seventh International Workshop on Polarized Sources and Targets (PST97), Urbana, IL, 1997, edited by R. J. Holt and M. A. Miller, [AIP Conf. Proc. 421, 337 (1997].

${ }^{3}$ T. Wise et al., Phys. Rev. Lett. 87, 042701-1 (2001).

${ }^{4}$ R. Engels et al., Rev. Sci. Instrum. 74, 4607 (2003).

${ }^{5}$ F. Rathmann et al., Proceedings of the 15th International Spin Physics Symposium, Upton, NY, 2002, edited by Y. I. Makdisi, A. U. Luccio, and W. W. MacKay, [AIP Conf. Proc. 675, 924 (2003)].

${ }^{6}$ H. F. Glavish, Nucl. Instrum. Methods 65, 1 (1968).

${ }^{7}$ Model C 500-MK5-St707, SAES Getters S. p. A, 20020 Lainate, Milano, Italy; German distributor SAES Getters, Deutschland, GmbH, D-50937 Köln, Germany; http://www. saesgetters. com.
${ }^{8}$ Sealed, free-end, single-core heater elements SEI $20 / 50-\mathrm{KT}=8 \mathrm{~cm}$, Thermocoax GmbH, D-22145 Stapelfeld, Germany.

${ }^{9}$ Type K, model 2ABI20/400 mm/TI/MF9F, Thermocoax GmbH, D-22145 Stapelfeld, Germany.

${ }^{10}$ NTN 1400-65, F.u.G. Elektronik GmbH, D-83024 Rosenheim, Germany.

${ }^{11} \mathrm{H}$. Kleines et al. (unpublished).

${ }^{12}$ HPT 100, Pfeiffer Vacuum GmbH, D-35614 Asslar, Germany.

${ }^{13}$ G. H. Dunn and L. J. Kiefer, Phys. Rev. 132, 2109 (1963).

${ }^{14}$ W. Bleakney, Phys. Rev. 35, 1180 (1930).

${ }^{15}$ D. Rapp et al., J. Chem. Phys. 42, 4081 (1965).

${ }^{16}$ Y. K. Kim et al., Electron-impact cross section database, 2002; http:// physics.nist.gov/PhysRefData/Ionization.

${ }^{17}$ MASSTORR DX, VG Quadrupoles Ltd., Middlewich, Cheshire, England. 